\title{
SUCCENTURIATE PLACENTA: AN INCIDENTAL FINDING DURING CESAREAN SECTION
}

\author{
Unmesh $^{1}$, Bhavya², S. Rudra ${ }^{3}$, Shivam ${ }^{4}$, Disha $^{5}$
}

1 Professor, Department of Obstetrics and Gynaecology, MMIMSR.

${ }^{2}$ Resident, Department of Obstetrics and Gynaecology, MMIMSR.

${ }^{3} \mathrm{HOD}$, Department of Obstetrics and Gynaecology, MMIMSR.

${ }^{4}$ Resident, Department of Microbiology, MMIMSR.

${ }^{5}$ Resident, Department of Obstetrics and Gynaecology, MMIMSR

ABSTRACT

A 30 years, 4th Gravida with 3 abortions with history of 8 months amenorrhea was admitted to the hospital with chief complaints of leaking per vagina since 4 hours and was not associated with pain abdomen or bleeding per vaginum. Perceiving decreased fetal movements since 6 hours. She had 3 previous missed abortions followed by D and E. In the present pregnancy, gestational age was 32 weeks at the time of admission.

Patient's general condition was stable, all other investigation were found to be normal her pulse was 100 beats/min, tachycardia present, BP -100/70 mmHg. On obstetric examination, uterus was 30 weeks size, 1-2 contraction lasting for 15- 20 seconds, Breech presentation, FHR was 124 per minute, regular, decreased liquor clinically. On vulvovaginal examination- Frank leaking per vagina present.

Ultrasound showed single live intrauterine pregnancy of 29 weeks 3 days with breech presentation with oligohydramnios, Placenta was at fundal region, Biophysical profile was 6/8, FHR $124 \mathrm{bpm}$.

After taking high risk consent in view of fetal prematurity, patient was posted for Emergency LSCS. And extracted a single live preterm female baby of weight of $1.45 \mathrm{~kg}$ by breech.

During the cesarean section, on opening abdomen lower segment was found to be congested with torturous vessels. So we suspected missed diagnosis of placenta previa. After delivery of the baby, we found succenturiate lobe of the placenta occupying lower uterine segment with vessels running across the membrane.

\section{KEYWORDS}

Succenturiate Placenta, Maternal Fetal Complication, Incidence.

HOW TO CITE THIS ARTICLE: Unmesh, Bhavya, Rudra S, et al. Succenturiate placenta: An incidental finding during cesarean section." Journal of Evolution of Medical and Dental Sciences 2015; Vol. 4, Issue 105, December 31; Page: 17086-17087, DOI: $10.14260 /$ jemds/2015/2590

\section{INTRODUCTION}

The succenturiate placenta is a morphological abnormality, one or more small accessory lobes connected to the main part of the placenta by blood vessels.[1] Incidence is about $3 \%$ $5 \% \cdot{ }^{[2]}$

\section{CASE REPORT}

A 30 years, 4th Gravida with 3 abortions with history of 8 months amenorrhea was admitted to the hospital with chief complaints of leaking per vagina since 4 hours and was not associated with pain abdomen or bleeding per vaginum. Perceiving decreased fetal movements since 6 hours. She had 3 previous missed abortions followed by D and E. In the present pregnancy, gestational age was 32 weeks at the time of admission.

Patient's general condition was stable, all other investigation were found to be normal her pulse was 100 beats/min, tachycardia present, BP-100/70mmHg. On obstetric examination, uterus was 30 weeks size, 1-2 contraction lasting for 15-20 seconds, Breech presentation, FHR was 124 per minute, regular, decreased liquor clinically.

Financial or Other, Competing Interest: None.

Submission 06-11-2015, Peer Review 07-11-2015,

Acceptance 01-12-2015, Published 31-12-2015.

Corresponding Author:

Dr. Bhavya,

\#61, Sukhdev Nagar,

Panipat,

Haryana.

E-mail: bhavyamaini@gmail.com

DOI:10.14260/jemds/2015/2590
On vulvovaginal examination-Frank leaking per vagina present. Ultrasound showed single live intrauterine pregnancy of 29 weeks 3 days with breech presentation with oligohydramnios, placenta was at fundal region, Biophysical profile was 6/8, FHR $124 \mathrm{bpm}$.

After taking high risk consent in view of fetal prematurity, patient was posted for Emergency LSCS and extracted a single live preterm female baby of weight of $1.45 \mathrm{~kg}$ by breech.

During the cesarean section, on opening abdomen lower segment was found to be congested with torturous vessels. So we suspected missed diagnosis of placenta previa. After delivery of the baby, we found succenturiate lobe of the placenta occupying lower uterine segment with vessels running across the membrane (Fig. 1), (Fig. 2).

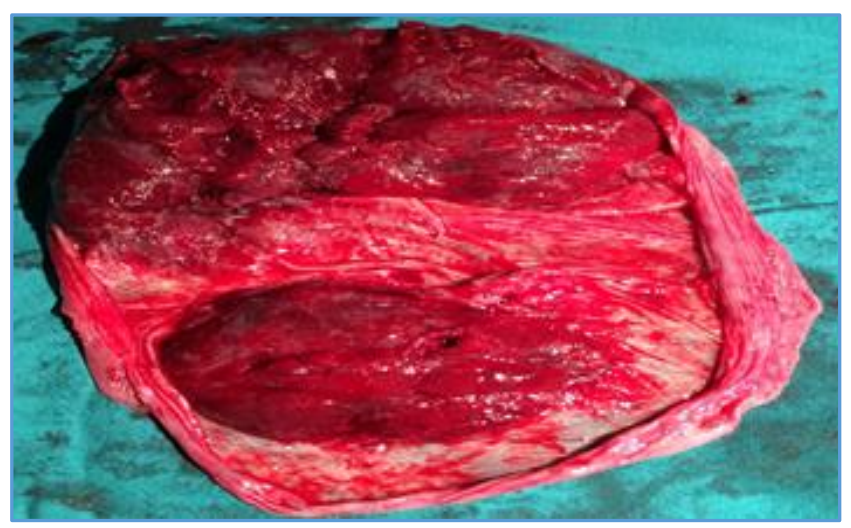

Fig. 1: Placenta with its two lobes 


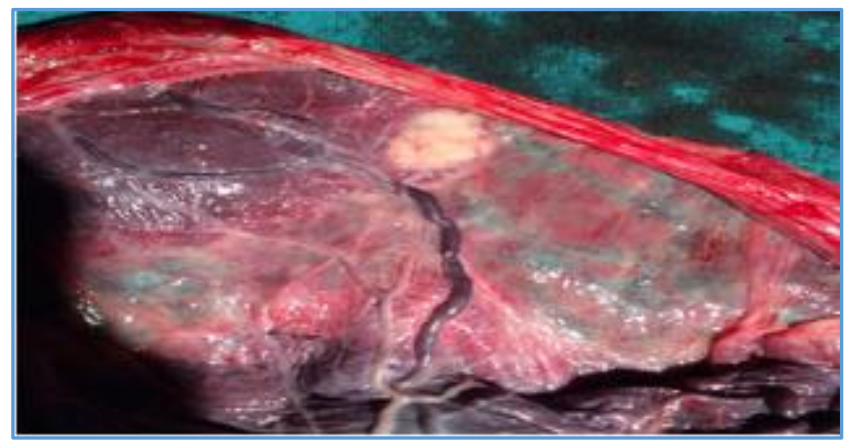

\section{Fig. 2: Succenturiate lobe of placenta showing vessels across the membranes}

This was an accidental finding during cesarean section. This type of the abnormality of the placenta are often associated with reduced placental efficiency and often leading to placental and consequently fetal growth impairment. It is usually noted that abnormally shaped placentae are more often associated with abnormal obstetric history and outcomes.(3)

\section{DISCUSSION}

The term succenturiate is derived from Latin word "succenturio" meaning "to substitute." Succenturiate lobes of the placenta result from focal areas of non-involution of the chorionic laeve. One or more accessory lobes are developed in the membranes at a distance from the periphery of the main placental mass. Vascular connections of the fetal origin usually connect the main placental mass to the succenturiate lobe. These accessory lobes may be single or multiple of varying size.

Placental abnormalities are an uncommon obstetric finding and among them the succenturiate lobe of placenta is a very rare entity which is common in elderly pregnant women aged more than thirty five. It is encountered mostly in complicated pregnancies which might result in fetal death. ${ }^{[4]}$ This rare entity is usually missed by trans-abdominal ultrasound.[5] In singleton pregnancies, the incidence of placental complications such as placental abruption, vasa previa and retained placenta were observed to be associated with the presence of abnormally shaped placentae.[6]

In gestational diabetes mellitus the incidence is very high so as to show $50 \%$ of the cases of succenturiate placenta may have a diabetic background, other common finding was the higher incidence of preterm labour.(3) probably the pathogenesis of this type of shape changes may be due to diabetes affecting the chorionic frondosum of the fetal part of the placenta and the macrosomia affecting the fetus. Other abnormalities that may be seen are vasa previa, retained succenturiate lobe, PPH and APH.(3) It will be too premature to conclude that succenturiate lobe of placenta is increased in incidence with ART as found by Suzuki and Igarashi.(7)

Another hypothesis is, malnourished mothers may have high incidence of abnormal morphology. In our case probably the previous history of 3 missed abortions and present premature delivery due to premature rupture of membranes may also be the result of same pathological process which has given rise to abnormality of placenta.

This case is a unique presentation of a succenturiate lobe of placenta in an elderly pregnant women which remained undiagnosed even after periodic antenatal ultrasound examinations of the placenta.

\section{CONCLUSION}

The succenturiate placenta is a morphological abnormality, the ultrasonography guided recognition, which in the antenatal period is important. This is due to the fact that the vessels connecting the main placenta with the succenturiate lobe may rupture during labour and lead to fetal demise. Though succenturiate placenta occurs rarely it should be kept as a differential diagnosis for Antepartum haemorrhage. Other accompanying complications are secondary $\mathrm{PPH}$, uterine sepsis, sub-involution are important. So sonography should be very thorough.

\section{REFERENCES}

1. Jeanty P, Kirkpatrick C, Verhoogen C, et al.; The Succenturiate Placenta; J Ultrasound Med 1983;2:9-12.

2. Dutta DC, Konar H (eds) abnormality of placenta and cord. $8^{\text {th }}$ ed New Delhi: Jaypee Brothers Medical Publishers (P) Ltd; 2015. p251.

3. Sudha R. Study of shape of placenta. National Journal of Basic Medical Science: vol II (4)1-5.

4. Suzuki S, Igarashi M. Clinical significance of pregnancies with succenturiate lobes of placenta. Arch Gynecol Obstet 2008 Apr;227(4):299-301.

5. Seleye-Fubara D, Akani CI. Succenturiate placenta: A rare variant in Rivers State Nigeria (A report of one case). Niger J med 2005 Jul-Sep;14(3):325-6.

6. Shukunami K, Tsunezawa W, Hosokowa K, et al. Placenta previa of a succenturiate lobe: A report of two cases. Eur J Obstet Gynecol Repord Biol 2001 Dec 1;99(2):276-7.

7. Suzuki S, Igarashi M, Inde $Y$, et al. Abnormally shaped placenta in twin pregnancy. Arch Gynecol Obstet 2010 Jan; 281(1):65-9. 